\title{
LETRAMENTO FUNCIONAL EM SAÚDE DE PESSOAS IDOSAS EM UMA UNIDADE DE SAÚDE DA FAMÍLIA
}

\section{FUNCTIONAL LITERACY ON HEALTH OF ELDERLY PEOPLE IN A FAMILY HEALTH UNIT}

\section{ALFABETIZACIÓN FUNCIONAL EN SALUd DE PERSONAS IDOSAS EN UNA UNIDAD DE SALUD DE LA FAMILIA}

Nidia Farias Fernandes Martins ${ }^{1}$, Daiane Porto Gautério Abreu ${ }^{2}$, Bárbara Tarouco Silva ${ }^{2}$, Eliel de Oliveira Bandeira ${ }^{3}$, Juliana Piveta de Lima $^{4}$, Julia Moraes Mendes 5 .

\begin{abstract}
RESUMO
Objetivo: analisar o Letramento Funcional em Saúde de pessoas idosas atendidas na Estratégia Saúde da Família, a partir do modo como elas buscam, compreendem e partilham informações em saúde. Método: Estudo qualitativo, exploratório-descritivo, com 23 pessoas idosas adscritas a uma Unidade de Saúde da Família. Foi utilizada a entrevista com um instrumento denominado "healthliteracy", adaptado e validado para o português. A análise dos dados realizou-se pela análise textual discursiva. Resultados: obtiveram-se quatro categorias: "busca por informações em saúde" - os participantes relataram a unidade como fonte de busca de informações, sentindo-se, em geral, satisfeitos com as informações; "compreensão das informações em saúde" - os participantes relataram que consideram as informações fáceis ou muito fáceis de entender; "compartilhamento das informações em saúde" - a maioria relatou compartilhar informações com familiares, amigos ou vizinhos; e "repercussões das informações em saúde" - as pessoas idosas consideraram que as informações fazem diferença em suas vidas, melhorando seu autocuidado, adesão a tratamentos, qualidade de vida, propiciando uma vida mais ativa. Conclusão: permite aos enfermeiros e demais profissionais da atenção básica saberem o modo de busca, compreensão e partilha de informações em saúde pelas pessoas idosas, planejando intervenções com utilizando a educação em saúde.
\end{abstract}

Descritores: Idoso; Alfabetização em saúde; Atenção primária à saúde; Estratégia saúde da família.

\begin{abstract}
Objective: to analyze the Functional Health Literacy of elderly people assisted in the Family Health Strategy, from the way they seek, understand and share health information. Method: A qualitative, exploratory-descriptive study with 23 elderly people enrolled in a Family Health Unit. The interview was used with an instrument called "health literacy", adapted and validated for Portuguese. The analysis of the data was carried out by the discursive textual analysis. Results: four categories were obtained: "search for health information" - the participants reported the unit as a source of information search, feeling generally satisfied with the information; "Understanding health information" - participants reported that they considered information easy or very easy to understand; "Sharing health information" - most reported sharing information with family, friends or neighbors; and "repercussions of health information" - the elderly considered that information makes a difference in their lives, improving their self-care, adherence to treatments, quality of life, providing a more active life. Conclusion: it allows nurses and other primary care professionals to know the way of searching, understanding and sharing health information by the elderly, planning interventions using health education.
\end{abstract}

Descriptors: Elderly; Health literacy; Primary health care; Family health strategy.

\section{RESUMEN}

Objetivo: analizarelLetramiento Funcional enSalud de personas ancianas atendidas enlaEstrategiaSalud de laFamilia, a partir del modo en que ellasbuscan, comprenden y comparteninformaciónensalud. Método: Estudiocualitativo, exploratorio-descriptivo, con 23 personas ancianasadscritas a una Unidad de Salud de laFamilia. Se utilizóla entrevista conun instrumento llamado "conocimiento sobre lasalud", adaptada y validada para elportugués. El análisis de losdatos se dio por elanálisis textual discursivo. Resultados: se obtuvieroncuatrocategorías: "búsqueda por informacionesensalud" - los participantes relataronlaunidad como fuente de búsqueda de informaciones, sintiéndose, en general, satisfechosconlasinformaciones; "comprensión de lasinformacionesensalud" - los participantes relataron que consideranlainformación fácil o muy fácil de entender; "compartir lainformaciónensalud" lamayoríarelató compartir informacionescon familiares, amigos o vecinos; y las "repercusiones de lasinformacionesensalud" - las personas mayoresconsideraron que lasinformacioneshacen diferencia en sus vidas, mejorandosu autocuidado, adhesión a tratamientos, calidad de vida, propiciando una vida más activa. Conclusión: permite a losenfermeros y demásprofesionales de laatención básica conocerel modo de búsqueda, comprensión y cómo compartir informaciónensalud por las personas ancianas, planeando intervenciones utilizando laeducaciónensalud.

Descriptores: Anciano; Alfabetización em salud; Atención primaria de salud; Estrategia de salud familiar.

${ }^{1}$ Graduada em Enfermagem. Doutoranda em Enfermagem da Universidade Federal do Rio Grande. ${ }^{2}$ Graduada em Enfermagem. Doutora em Enfermagem pela Universidade Federal do Rio Grande. Docente na Universidade Federal do Rio Grande. ${ }^{3}$ Graduado em Enfermagem. Mestrando em Enfermagem na Universidade Federal do Rio Grande. ${ }^{4}$ Graduada em Enfermagem. Doutoranda em Enfermagem pela Universidade Federal do Rio Grande. ${ }^{5}$ Graduanda em Enfermagem Universidade Federal do Rio Grande. 


\section{INTRODUÇÃO}

O envelhecimento populacional é um fenômeno mundial que vem crescendo significativamente. Estima-se que a proporção de pessoas idosas na população mundial de 2015 aumente de $12,3 \%$ para $24,6 \%$ em cerca de 55 anos. No Brasil, no ano de 2015 , a proporção de idosos foi de $11,7 \%$, bem próxima ao indicador mundial, e espera-se que irá dobrar, chegando ao percentual de $23,5 \%$ de idosos em 24 anos $^{(1)}$.

As mudanças no perfil epidemiológico da população brasileira aumentam a demanda por serviços para as pessoas idosas, principalmente na atenção primária à saúde, devido às doenças crônicas não transmissíveis e suas comorbidades, tornando-se um desafio para os profissionais de saúde que atuam nesse âmbito. Além disso, com as limitações advindas do processo de envelhecimento, as pessoas idosas, muitas vezes, apresentam dificuldade em seguir o tratamento de forma sistemática e regular, por não compreenderem adequadamente as orientações ofertadas pelos profissionais. Esse fato é considerado como um dos principais desafios na atenção à saúde, afetando os comportamentos de saúde ${ }^{(2)}$.

Nesse sentido, as ações de saúde voltadas para as pessoas idosas na atenção primária e na Estratégia Saúde da Família (ESF) são permeadas pela educação nessa área, visando o autocuidado, a independência e autonomia dessas pessoas ${ }^{(3)}$. Essas ações precisam ser desenvolvidas numa perspectiva dialógica, emancipadora, participativa, contribuindo para a autonomia da pessoa idosa no que se refere à sua trajetória de saúde e doença e autonomia dos profissionais diante da possibilidade de reinventar modos de cuidado mais humanizados, compartilhados e integrais ${ }^{(4)}$.

Entretanto, é preciso saber o quanto essas pessoas estão, de fato, assimilando (obtendo, processando e entendendo), empoderando-se e utilizando esse conhecimento, corretamente, em suas vidas. Isso se dará através do conhecimento do grau de Letramento Funcional em Saúde (LFS) dessa população específica ${ }^{(2)}$.

O LFS é definido como o grau no qual os indivíduos têm a capacidade para obter, processar e entender informações básicas, escritas ou faladas sobre saúde e serviços necessários para a tomada de decisões adequadas em saúde. Essa "capacidade" é mediada por fatores como a educação, a cultura, a linguagem, as crenças. $E$ quando se fala em "tomada de decisões adequadas em saúde", essa é mediada por fatores como a interação dos sujeitos nos ambientes de saúde, a influência da mídia e da política, do mercado, entre outros, levando em consideração tanto os fatores individuais como sociais ${ }^{(5)}$.

Nesse contexto, o LFS é um conceito relevante, principalmente nos aspectos que envolvem $o$ processo saúde-doença da população, pois essa abordagem exige a melhoria dos recursos que as pessoas necessitam para ter um posicionamento ativo frente às questões da própria saúde, dos seus familiares e da sua comunidade, incluindo, nesse processo, a capacidade de mudar as condições de saúde vigentes ${ }^{(2,6)}$.

Alguns grupos populacionais como o de pessoas idosas, com baixa renda e baixa escolaridade podem ser marginalizados em relação ao $\mathrm{LFS}^{(7)}$. Além disso, as pessoas com baixo grau de LFS têm grande dificuldade em realizar o autocuidado, ainda mais na presença de danos crônicos, resultando em baixa adesão a tratamentos, pouca participação nos serviços de saúde, internações hospitalares e mortalidade elevada e precoce, ocasionando também um aumento dos custos em saúde ${ }^{(6)}$.

O LFS é um tema amplamente abordado no cenário internacional. Nos Estados Unidos da América, estima-se que mais de um terço dos adultos tenham LFS limitado, o que torna mais difícil a leitura, compreensão e aplicação das informações em saúde ${ }^{(6)}$. Em um estudo de revisão sistemática e meta-análise realizado, observou-se que a idade avançada é fortemente associada ao limitado LFS, com maior probabilidade de as pessoas idosas terem LFS baixo ${ }^{(8)}$.

No Brasil, ainda é limitado o número de pesquisas que evidenciem o grau de LFS e se o fenômeno pode estar afetando o resultado de saúde da população. Apesar disso, alguns autores vêm trabalhando pontualmente com o tema e pode-se observar que a temática vem sendo cada vez mais desenvolvida no Brasil, com estudos mais recentes com idosos ${ }^{(9-10)}$, em que se percebe um percentual de até $68,1 \%$ de LFS inadequado.

0 baixo LFS tem consequências diretamente relacionadas com a saúde da população, como no envolvimento das pessoas em práticas de saúde preventiva, na detecção precoce de doenças, no gerenciamento das doenças crônicas e no acesso e utilização dos serviços de saúde ${ }^{(2)}$. Assim, percebe-se que o 
estudo sobre LFS em pessoas idosas é relevante, pela necessidade de ampliar os conhecimentos relacionados à essa temática, bem como contribuir para que enfermeiros e demais profissionais subsidiem as intervenções realizadas no que tange ao desenvolvimento de ações de educação em saúde, voltando os olhares às necessidades de busca, compreensão e compartilhamento das informações em saúde.

O estudo teve como objetivo analisar o LFS de pessoas idosas atendidas na ESF, a partir do modo como elas buscam, compreendem e partilham informações em saúde.

\section{MÉTODO}

Tratou-se de um estudo exploratório, descritivo, com abordagem qualitativa, realizado em uma Unidade de Saúde da Família (USF) localizada em um município do extremo Sul do Rio Grande do Sul.

Para a coleta dos dados, foi utilizado um instrumento desenvolvido por pesquisadores canadenses denominado healthliteracy ${ }^{(11)}$, adaptado transculturalmente e validado em português ${ }^{(12)}$. Ele é composto por questões abertas e fechadas, com o objetivo de avaliar como os idosos buscam, compreendem e partilham informações em saúde, baseadas por um interesse, preocupação ou situação de saúde vivenciada recentemente pelos entrevistados (no último mês, o que o(a) Sr. ${ }^{(a)}$ pensou sobre sua saúde?) escolhido para dar seguimento à entrevista.

As demais questões versavam sobre o entendimento do entrevistado sobre a situação de saúde escolhida e suas dúvidas a respeito da mesma, as fontes de informação utilizadas, sua satisfação, a utilidade, a compreensão, a coerência, o compartilhamento e o impacto das informações em sua vida. As questões fechadas avaliaram a satisfação e o entendimento das informações sobre saúde pelos idosos, utilizando Escala tipo Likert e reforçando as opiniões expressas nas questões abertas ${ }^{(12)}$.

Os participantes foram 23 idosos atendidos e adstritos à referida unidade no período de realização da pesquisa. Esses foram selecionados com base nos seguintes critérios: usuários com idade igual ou superior a 60 anos; e ser adstrito à área de abrangência da USF. Os critérios de exclusão foram: apresentar discurso desconexo com perdas importantes de memória que impeçam respostas às questões do instrumento de coleta de dados. Os participantes foram selecionados intencionalmente através de uma amostragem por conveniência. Neste estudo, optou-se por incluir como participantes apenas as pessoas idosas responsáveis pela gestão do seu regime terapêutico. Sabe-se que, no que se refere ao cuidado à saúde da pessoa idosa, muitas vezes, o cuidado é realizado em parceria com os cuidadores formais e informais. Em pesquisas futuras, sugere-se a inclusão de outros indivíduos que participam no cuidado à pessoa idosa.

A coleta de dados ocorreu de agosto a outubro de 2015, por meio de entrevistas que foram gravadas em um gravador de voz e transcritas posteriormente. Para garantir o anonimato e a confidencialidade, cada idoso foi identificado pela letra "I" seguida do número que corresponde à ordem de realização da entrevista. A entrevistadora foi a enfermeira autora principal deste artigo. O tempo médio de duração das entrevistas foi de 13 minutos.

Os idosos foram convidados para participar da pesquisa quando compareciam na USF para realizar algum tipo de atendimento, em seus domicílios, ou ainda durante as visitas domiciliares de rotina, com os agentes comunitários de saúde. As entrevistas foram realizadas em uma sala da USF, que foi destinada para esse fim ou no domicílio do idoso. Destacase que, por vezes, havia a presença de familiares junto à pessoa idosa que estava sendo entrevistada, sendo solicitado que não interferissem nas respostas da entrevista. Foi realizado um teste piloto com um idoso fora da área de abrangência da USF, no início de agosto de 2015, antes da realização da coleta de dados na USF, no qual se verificou a adequação da linguagem do instrumento.

Os dados foram analisados por meio da técnica de análise textual discursiva. Essa abordagem de análise pode ser concebida como um processo auto-organizado de produção de novas compreensões em relação aos fenômenos que examina ${ }^{(13)}$.

A análise proposta organiza argumentos em torno de quatro focos. Os três primeiros compõem um ciclo, no qual se constituem como elementos principais: desmontagem dos textos, também chamado de unitarização, em que são examinados os materiais e seus detalhes, fragmentando-os para atingir unidades de significado referentes ao fenômeno estudado; estabelecimento de relações ou categorização, onde reúnem-se as unidades de significado semelhantes, podendo gerar vários níveis de 
categorias de análise; captando o novo emergente, onde a intensa impregnação nos materiais da análise desencadeada pelo processo anterior possibilita uma compreensão renovada do todo, fechando o ciclo de análise. Por fim, o último foco, um processo auto-organizado em que, após a fragmentação e desorganização propostas na primeira fase, ocorre uma reconstrução com emergência de novas compreensões $^{(13)}$.

O projeto respeitou as normas de pesquisa envolvendo seres humanos, foi aprovado pelo comitê de ética local, sob o parecer no 61/2015. CAAE: 44623415.2.0000.5324. Foi fornecido termo de consentimento em duas vias para os participantes. Destaca-se que os participantes da pesquisa e profissionais da USF foram convidados para apresentação multimídia e explanação dos resultados da pesquisa, que foi realizada na própria USF, pelas autoras.

\section{RESULTADOS E DISCUSSÃO}

Entre os participantes do estudo, a maioria $(69,5 \%)$ era do sexo feminino, casada $(43,7 \%)$, e possuía entre um a quatro anos de estudos (56,5\%). A média de idade dos participantes foi de 68 anos, variando entre 60 e 85 anos.

Predominou-se a baixa escolaridade entre os idosos entrevistados, sendo a média verificada de 2,91 anos de estudos, variando em zero (analfabeto) e sete anos de estudo. Isto pode influenciar diretamente nos resultados do LFS, pois pessoas idosas com baixa escolaridade tendem a apresentar maior dificuldade no entendimento, obtenção e uso das informações em saúde, como evidenciado em outro estudo ${ }^{(7)}$.

A maioria dos idosos entrevistados declarou ter residência própria (91,3\%) e morar com esposo (a) $(43,4 \%)$ e com filhos (as) $(30,4 \%)$. A renda familiar mensal variou de um a dois salários mínimos, com média de 1,76 . A renda individual mensal dos idosos também variou de 1 a 2 salários mínimos, com média de 1,15. O valor do salário mínimo na época da coleta era de $\mathrm{R} \$$ 788,00.

A baixa renda e baixa escolaridade também foram evidenciadas em estudos sobre LFS e elas contribuem para dificuldade de acesso à informação e aos serviços de saúde ${ }^{(9)}$.

Os principais interesses ou preocupações de saúde vivenciadas pelos idosos foram relacionados à doenças crônicas, relatados por 17 idosos $(73,9 \%)$ ou sinais e sintomas de doenças, relatados por nove idosos $(39,1 \%)$, sendo semelhantes aos do estudo canadense ${ }^{(11)}$. Para dar seguimento à entrevista, a maioria dos idosos (15-65,2\%) escolheu alguma situação dessa temática.

Os participantes foram questionados, por último, se participavam de algum grupo de educação em saúde na USF, e sete $(30,4 \%)$ disseram participar. O grupo tem frequência mensal e, dos que declararam participar do grupo, a participação variou entre 1 a 6 anos, tendo como média 3,5 anos de participação. As práticas desenvolvidas no grupo de educação em saúde nas unidades básicas de saúde, geralmente, são práticas bem pontuais acerca de orientações sobre a patologia, esquema terapêutico prescrito e estilo de vida saudável.

Para alcançar o objetivo do estudo, os resultados estão organizados em quatro categorias a priori, seguindo o roteiro do instrumento de coleta de dados. São elas: "busca por informações em saúde"; "compreensão das informações em saúde"; "compartilhamento das informações em saúde"; e "repercussões das informações em saúde".

\section{Categoria 1 - Busca por informações em saúde}

Os idosos relataram diversas dúvidas sobre a situação de saúde vivenciada, o que mostra que não foram esclarecidos, não compreenderam as informações fornecidas ou não procuraram informações que as esgotassem. As principais dúvidas foram em relação a tratamento e cuidados para manter-se saudável e reduzir os danos das doenças crônicas (12 idosos), sobre as repercussões que poderiam afetar as suas vidas (seis idosos), sobre a fisiopatologia e causas (cinco idosos), e sinais e sintomas das doenças (quatro idosos). Outros quatro idosos não tinham nenhuma dúvida sobre a situação de saúde escolhida.

Um estudo realizado na África do Sul que investigou as necessidades e comportamentos de busca de informações sobre medicamentos, com usuários na atenção primária mostrou que os participantes, apesar de terem dúvidas ou demonstrarem conhecimento insuficiente relacionado aos medicamentos, muitas vezes, não procuram informações ou não questionam os profissionais por, simplesmente, não saberem da possibilidade de que poderiam perguntar, ou por não serem encorajados a fazê-lo ${ }^{(14)}$.

O encorajamento e o incentivo da procura de informações de saúde e no questionamento de suas dúvidas podem ser estimulados para que 
haja o empoderamento da pessoa idosa nas suas questões de saúde e doença, gerando melhores resultados de saúde ${ }^{(14)}$.

Nesse sentido, os profissionais podem focalizar a educação em saúde nas reais necessidades dos clientes, nas suas dúvidas e no seu contexto social e cultural e, não somente, no que o profissional acha importante para a pessoa idosa.

Os idosos citaram, como primeira fonte para a busca de informações em saúde, a USF (12 idosos), seguido de hospitais (quatro idosos) e consultórios de especialistas (quatro idosos). Uma idosa citou, como primeira fonte de informação, a televisão. Os profissionais mais procurados foram, prioritariamente, médicos, seguidos de enfermeiros, técnicos de enfermagem e odontólogos.

A busca por profissionais de saúde, tendo como prioridade, médicos e enfermeiros como fonte de informação, também foi relatado em outros estudos $^{(9,14)}$. A busca por profissionais é um elemento que remete à confiança, pois esses foram preparados para atender às questões de saúde, o que é, geralmente, relacionado à experiência e ao estudo desses profissionais ${ }^{(15)}$. Esse resultado reforça a importância da comunicação efetiva entre profissionais e usuários, pois essa é uma das fontes mais procuradas $^{(16)}$.

Outras fontes de busca de informação menos citadas, porém consideradas como de importante destaque para os idosos entrevistados, foram os meios de comunicação como a internet e a televisão, pela diversidade de informações expostas, o que evidencia que, apesar da baixa escolaridade dos idosos que participaram do estudo, estes estão procurando alternativas modernas, como a inclusão digital. No estudo canadense ${ }^{(11)}$, que criou e utilizou o instrumento da presente pesquisa, os meios de comunicação foram mais utilizados. Em um outro estudo realizado em Londres, na Inglaterra, mostrou que o uso da internet e o engajamento social ajudam os idosos a manter o LFS eficiente durante o envelhecimento ${ }^{(17)}$.

Os idosos, em geral, sentiram-se satisfeitos ou muito satisfeitos com as informações recebidas (19 idosos, com a primeira fonte, e 13 idosos, com as demais fontes). Apenas dois idosos sentiram-se neutros. Não houve insatisfação com as fontes de informação.

Alguns idosos destacaram terem sido bem esclarecidos e colocaram o bom atendimento e tratamento como um requisito básico na busca de informações em saúde. Outros idosos, em sua maioria, os que citaram como fonte de informação a USF, relataram a confiança e o vínculo com a USF e com os profissionais que lá atuam, como importante na busca por informações de saúde, como evidenciam as falas: "[...] não me mudo daqui por causa do posto [...]. Todos enfermeiros gente boa, me tratam com bastante carinho, todos dali do posto, a médica, qualquer um que eu chego ali, tudo me trata bem [...]" (I 23)

"[...] Eu só vou aqui! Me acostumei aqui! [...]" (। 16)

A principal fonte de informação requisitada pelos idosos foi a USF estudada, o que mostra o diferencial da ESF na vida desses idosos, que demonstraram satisfação, confiança, facilidade de acesso e vínculo com a unidade.

O vínculo é previsto na atenção básica como um suporte no acompanhamento das pessoas idosas. Seu diferencial na saúde da família se dá por meio da atenção continuada e visitas domiciliares, onde os profissionais poderão conhecer e perceber as especificidades desses idosos, adentrar em sua realidade, propiciando com isso que o idoso se sinta mais à vontade e possa aderir às intervenções propostas pela equipe de saúde.

Para o entendimento do LFS dos idosos na atenção básica, o vínculo exerce importante papel, à medida que os profissionais têm mais conhecimento e liberdade para trabalhar com essa população ${ }^{(18)}$.

Como fonte mais útil, 15 idosos citaram a USF, três idosos, consultórios de especialistas e quatro idosos não diferenciaram fonte mais útil. Dos que citaram, como fonte útil, os especialistas, não haviam referido a USF como fonte de informação. Entre as fontes que confiam mais, a USF também foi a mais citada (15 idosos). Apenas três idosos citaram especialistas e também não haviam referido a USF como fonte de informação.

Alguns dos que citaram a USF como fonte mais útil e confiável, referiam-se aos profissionais atuantes no local e também ao vínculo, à facilidade de acesso à unidade, à continuidade do atendimento, e ao acompanhamento através da realização de consultas, grupos e visitas domiciliares pela equipe de saúde.

Uma idosa citou também o aspecto econômico como utilidade, pois adquire medicamentos e material de curativo na 
unidade. Também foi destacado, nas falas, a possibilidade de acolhimento, escuta e troca, o que não é possível com fontes de informação não humanas.

"[...] ela já sabe onde eu moro e tudo [...]" (I 02) "[...] eu confio muito nessa doutora aqui [...]. Ela vem periodicamente aqui, nas quintas feiras, então uma pessoa que está mais acostumada comigo, e eu estou acostumado com ela, então eu confio mais nela [...]"

"[...] a gente conversando direto, faz a diferença! [...] porque a gente expõe o que a gente sente, tudo, e a doutora explica tudo, tem o grupo lá para atender, eu acho ótimo lá! [...]" (I 06)

Percebe-se que a facilidade de acesso e o vínculo são fatores importantes na busca das informações em saúde e que influencia no LFS dessa população, pois se sentem mais à vontade para buscar a unidade como uma fonte confiável na qual conseguirão respostas para sua busca ${ }^{(19)}$. Desse resultado, emerge uma potencialidade a ser trabalhada pelos profissionais de saúde na ESF pois, através da confiança e do vínculo, podem buscar melhorias na busca e no entendimento de informações de saúde pelas pessoas idosas no seu cotidiano de trabalho.

\section{Categoria 2 - Compreensão das informações em saúde}

Em relação ao nível de entendimento das informações, a maioria dos idosos (18) considerou-as fácil ou muito fácil de entender. Um idoso considerou como neutro e outros três idosos consideraram como difícil. Alguns ainda destacaram o interesse próprio como aliado no entendimento das informações:

"[...] são fáceis, simplesmente vai depender da gente, do interesse. [...]" (I 04)

"[...] eu não achei dificuldade porque eu acho que eu estou fazendo a minha parte [...]" (I 14)

"[...] é fácil, só ter vontade [...]" (I 15)

Percebe-se que os idosos consideram a importância do interesse próprio na compreensão das informações, por exemplo, quando ouviam palavras que não sabiam 0 significado. Tal fato demonstra a autonomia dos idosos em suas decisões, na busca e no entendimento das informações de saúde.

Eles percebem que a iniciativa não deve ser somente do profissional de saúde, mas que eles também são protagonistas no seu cuidado. $O$ incentivo da autonomia e independência do idoso também deve ser estimulado pelos profissionais da atenção básica, como um aliado na promoção e recuperação da saúde dessa parcela da população, o que é preconizado pela Política Nacional de Atenção Básica ${ }^{(18)}$.

Quando questionados se havia discordância entre as informações, a maioria (20) respondeu que não; porém, um entrevistado referiu discordância em informações da internet, que muitas vezes podem não ser confiáveis.

"[...] eu acho que o médico estava mais certo, porque não ligou um com o outro, sabe, o que a internet diz e o que o médico fala [...]" (I 09)

Ao serem questionados se, alguma vez, ouviram palavras que não entenderam, ou não sabiam o significado, 10 dos entrevistados disseram nunca ter ouvido. Oito dos idosos ouviram, ocasionalmente, e três, frequentemente. Diante dessa situação, a maioria (nove) perguntou diretamente para a pessoa ou para familiares.

O uso de termos médicos específicos e linguagem incompatível tem sido descrito como uma grande barreira na comunicação entre profissionais e usuários da atenção primária, o que compromete a compreensão das informações por parte dos usuários, afetando seu $\mathrm{LFS}^{(20)}$.

\section{Categoria 3 - Compartilhamento das informações em saúde}

Os idosos relataram, em sua maioria, ter compartilhado a sua preocupação ou interesse e as informações recebidas com os familiares (10 idosos), seguido dos amigos, vizinhos ou conhecidos (oito idosos), e também com os profissionais de saúde (seis idosos). Sete idosos preferiram não compartilhar com ninguém. Compartilhar preocupações, principalmente com a família, é uma forma de obter ajuda na tomada de decisões, obter apoio e expressar sentimentos (medo, mágoa, insegurança) como forma de alívio, de confiança e vínculo com a pessoa que está compartilhando.

"[...] converso mais com os filhos, em quem a gente confia mais [...]" (I 09)

"[...] com o pai, com a minha irmã, com quem eu fico mais junto [...]" (I 04)

"[...] foi bom que ela me ajudou, ela disse, mas tu tens que seguir o que é [...]" (I 15)

"[...] a gente tem que dar força para os outros [...]" (I 19)

O compartilhamento das informações também propicia que haja a transmissão de conhecimentos e aprendizados para outras 
pessoas, tornando-se multiplicadores de saúde e levando conscientização a outros idosos.

"[...] os cuidados, eu já até passei os cuidados, o médico passou pra mim eu já passo pra outros [...]" (I 09)

"[...] conversei com uma senhora, que era diabética também, [...] ela também gostava de um baile, de uma cerveja, ela disse que parou e está se sentindo muito bem! [...]" (I 22)

A partilha das informações com familiares, amigos ou vizinhos, também foi frequente nos estudos que utilizaram o mesmo instrumento ${ }^{(9,21)}$. O papel da família, amigos e vizinhos como rede de apoio informal na vida da pessoa idosa é crucial para o suporte da saúde e tomada de decisões. A existência de redes de apoio social é um importante elemento no compartilhamento de preocupações e situações para os idosos, e tem sido relatado como interagindo e influenciando no LFS $^{(22)}$.

A influência dessas redes de apoio no LFS de pessoas idosas é visível no presente estudo e deve ser estimulada na ESF, por meio do incentivo à participação da família e da comunidade nas ações educativas. Esse compartilhamento de informações possibilita a troca de conhecimentos e a execução de práticas favoráveis à saúde ${ }^{(3)}$.

Quando questionados sobre o que seria mais importante para outros idosos saberem, em relação à situação de saúde escolhida, 13 idosos relataram formas de modificar o estilo de vida e comportamentos, expressas em mudanças de hábitos como cessar o fumo e álcool, aderir à alimentação saudável e prática de atividade física, entre outros, como é expresso nas falas:

"[...] é não fumar e não beber [...]" (I 21)

"[...] que ninguém fume, isso aí é o conselho que eu dou [...]" (I 11)

"[...] é o exercício e a alimentação [...]" (I 06)

"[...] não comer sal, tomar bastante água, comer bastante fruta, verduras [...]" (I 04)

Outro aspecto considerado como importante para outras pessoas idosas saberem foi ter uma compreensão mais ampliada da doença, abordando as causas, tratamentos e cuidados para evitar o agravamento, destacado por 11 idosos, como é expresso nas falas:

"[...] te cuida, não pega frio, que é para não pegar doença [...]" (I 17)

"[...] é saber se cuidar, tomar remédio direitinho [...]" (I 19)

Esses resultados vão ao encontro dos achados de estudo que utilizou o mesmo instrumento ${ }^{(21)}$, e atentam para a importância de os profissionais focarem na assistência, não somente nas questões relativas às doenças, mas à promoção da saúde e de outros aspectos relacionados, com foco no envelhecimento ativo e saudável ${ }^{(23)}$.

\section{Categoria 4 - Repercussões das informações em saúde}

Quase a totalidade dos idosos (21) reconhece que as informações de saúde que foram buscadas, recebidas ou compartilhadas, fizeram diferença nas suas vidas. A grande parte dos idosos destacou que as informações melhoraram seu autocuidado e a adesão a tratamentos:

"[...] vou procurar me cuidar mais, que é para não passar pelo mesmo problema, não é? [...]" (I 17)

"[...] mas aí eu parei com a comida [...] comecei a tomar os remédios [...]" (1 22)

"[...] eu me cuido mais [...] eu sei que eu não posso fazer, eu já não faço [...]" (I 11)

Os idosos destacaram ainda que, com as informações recebidas, puderam ter uma melhor qualidade de vida e bem-estar, adquirir novos conhecimentos e ter uma vida mais ativa, com autonomia e independência, e puderam aceitar melhor a sua condição de saúde.

"[...] me sinto melhor hoje [...]" (I 09)

"[...] coisas que eu não entendia [...] aqui dentro do posto médico eu pergunto, eu fiquei sabendo de coisas que eu não sabia, que eu não tinha conhecimentos [...]" (। 14)

"[...] nunca estar parado! [...]" (I 21)

Quanto a essas repercussões, percebeuse que trouxeram benefícios e mudanças à vida dos idosos e que envelhecer saudável era possível através da obtenção dessas informações sobre saúde. Essas repercussões citadas provam que o trabalhar com o LFS dessa população é possível de diversas maneiras na atenção básica como em consultas, visitas domiciliares, grupos, entre outros. Na prática de promoção da saúde, o LFS significa compreender as condições que determinam a saúde e saber como mudá$\operatorname{las}^{(24)}$.

Quando questionados sobre a repercussão do grupo de educação em saúde em suas vidas, os idosos relataram que a participação no grupo propicia a eles o bem-estar, a possibilidade de distração, fazer novas amizades, o estabelecimento de vínculos e intimidades e a troca de conhecimentos. Também para 
continuarem uma vida ativa como relatam, desejando que o grupo tenha encontros mais frequentes e com práticas de atividades físicas variadas, esperando que o grupo continue cada vez mais forte na USF.

"[...] a gente adquire mais experiência, tem mais conhecimento [...]" (I 04)

"[...] se pudesse ter mais seguido, e ter exercícios [...]" (I 13)

"[...] eu me sinto bem, eu me sinto na minha casa [...]" (I 14)

"[...] eu vou, para escutar, para ver o que elas vão ensinar para gente [...]" (I 03)

Esses relatos demonstram a importância dos grupos de convivência, permitindo que a pessoa idosa se mantenha ativa e incluída nas atividades sociais, promovendo troca de experiências e melhorando sua auto-estima e qualidade de vida. Além disso, diminui preconceitos dos idosos consigo mesmo, favorece a adesão aos tratamentos, e cria vínculo entre a unidade e a família do idoso, tornando-se também uma rede de apoio e troca de vivências ${ }^{(25)}$. Portanto, os grupos também despontam como uma potencial ferramenta para trabalhar diversas questões envolvidas no LFS.

Uma das limitações desse estudo é que os resultados não podem ser generalizados, visto que abrange um local e uma parcela específica de usuários da ESF. Outra limitação é que o instrumento utilizado impossibilita a avaliação de um "grau" de LFS, como a maioria dos instrumentos utilizados; porém fornece uma análise mais qualitativa, que pode ser considerada um viés, visto que a avaliação do grau de LFS poderia ter um resultado diferente do descrito no presente estudo.

\section{CONCLUSÃO}

O estudo permitiu analisar o LFS, considerando a trajetória desses idosos na atenção básica e na ESF. Em uma concepção ampliada, foi possível saber o modo como as pessoas idosas estão captando informações para sua saúde e isso se relaciona à facilidade de acesso e o vínculo que os participantes têm com a USF estudada, o que é fundamental na busca, no entendimento, no compartilhamento e nas repercussões das informações de saúde importantes para esses idosos.

0 método qualitativo utilizado neste estudo através do instrumento de coleta de dados permitiu um caminho mais viável para análise do LFS, pois não abrange habilidades de leitura e de escrita, como na maior parte dos instrumentos utilizados, mas sim uma história e uma trajetória de como os idosos buscam, compreendem e partilham informações de saúde em suas vidas, atingindo assim o objetivo proposto.

O estudo permite aos enfermeiros e demais profissionais que atuam na atenção básica, saber como conhecer o LFS de idosos, saber o que os preocupa, como eles buscam, compreendem e partilham informações de saúde e, a partir disso, planejar e desenvolver ações ou intervenções focalizadas, através de uma comunicação adequada com o usuário e captação de suas necessidades e questionamentos, possibilitando o estímulo ao autocuidado e o correto seguimento de tratamentos de saúde. Destaca-se a ferramenta da educação em saúde, que permeia todas as ações desenvolvidas, e a sensibilização para a avaliação das reais necessidades de conhecimento das pessoas idosas, levando em conta suas especificidades e experiências.

\section{REFERÊNCIAS}

1- Instituto Brasileiro de Geografia e Estatística (IBGE). Síntese de indicadores sociais: Uma análise das condições de vida da população brasileira. Rio de Janeiro: IBGE; 2016.

2-Passamai MPB, Sampaio HAC, Lima JWO. Letramento funcional em saúde de adultos no contexto do Sistema Único de Saúde. Fortaleza: EdUECE; 2013.

3-Gautério DP, Vidal DAS, Barlem JGT, Santos SSC. Action by nurses to educate older adults: The family health strategy. Rev Enferm UERJ 2013 [citado 2017 mar 25]; 21(nesp 2):824-8. Disponível em: http://www.e-publicacoes.ueri.br/ index.php/ enfermagemueri/article/view/12302

4-Brasil. Ministério da Saúde. II Caderno de educação popular e saúde. Brasília, DF: Ministério da Saúde; 2014.

5- Institute of Medicine (IOM). Health literacy: A prescription to end confusion. Washington, DC: National Academies Press; 2004.

6- Hersh L, Salzman B, Snyderman D. Health literacy in primary care practice. Am Fam Physician 2015 [citadoem 25 mar 2017]; 92(2):118-24. Disponívelem: http://www.aafp.org/afp/2015/0715/p118.html

7- Liu Y-B, Liu L, Li Y-F, Chen Y-L. Relationship between Health Literacy, Health-Related Behaviors and Health Status: A survey of elderly 
Chinese. Int J Environ Res Public Health 2015; 12(8):9714-25. DOI: 10.3390/ijerph120809714 8- Kobayashi LC, Wardle J, Wolf MS, Wagner CV. Aging and functional health literacy: A systematic review and meta-analysis. J Gerontol B Psychol Sci Soc Sci. 2016;71(3):445-457. DOI: 10.1093/geronb/gbu161

9- Machado ALG, Gubert FA, Pinheiro PNC, Vieira NFC, Oliveira PS, Guedes IH. Letramento em saúde e envelhecimento: Foco em condições crônicas de saúde.In: Atas da $3^{\circ}$ Congresso IberoAmericano de Investigação Qualitativa,Espanha. Espanha: CIAIQ;2015. p. 187-92.

10- Santos MIPO, Portella MR, Scortegagna HM, Santos PCS. Functional health literacy from the perspective of gerontological nursing: An integrative literature review. RevBrasGeriatrGerontol.2015;18(3):651-64. DOI: 10.1590/1809-9823.2015.14080

11- Kwan B, Frankish J, Rootman I. The development and validation of measures of health literacy in different populations. Columbia: University of British Columbia; 2006.

12-Paskulin LMG, Aires M, Valer DB, Morais EP, Freitas IBA. Adaptation of an instrument to measure health literacy of older people. Acta Paul Enferm.2011;24(2):271-7. DOI: 10.1590/S010321002011000200018

13- Moraes R, Galiazzi MC.Análise textual: Discursiva. 2a ed. Ijuí: Editora Unijuí; 2011.

14- Patel S, Dowse R. Understanding the medicines information-seeking behaviour and information needs of South African long-term patients with limited literacy skills. Health Expect. 2015;18(5):1494-507. DOI: 10.1111/hex.12131

15- Clarke MA, Moore JL, Steege LM, Koopman RJ, Belden JL, Canfield SM, et al. Health information needs, sources, and barriers of primary care patients to achieve patient-centered care: A literature review. Health Informatics J. 2015;22(4):992-1016.

DOI:

\section{$10.1177 / 1460458215602939$}

16- Gutierrez N, Kindratt TB, Pagels P, Foster B, Gimpel NE. Health literacy, health information seeking behaviors and internet use among patients attending a private and public clinic in the same geographic area.J Community Health 2014;39(1):83-9. DOI: 10.1007/s10900-013-97425

17-Kobayashi LC,Wardle J,Von Wagner C. Internet use, social engagement and health literacy decline during ageing in a longitudinal cohort of older english adults. J Epidemiol Community
Health 2015;69(3):278-83. DOI: 10.1136/jech2014-204733

18-Brasil. Ministério da Saúde. Portaria no 2.436, de 21 de setembro de 2017. Aprova a Política Nacional de Atenção Básica, estabelecendo a revisão de diretrizes para a organização da Atenção Básica, no âmbito do Sistema Único de Saúde (SUS). Brasília, DF: Ministério da Saúde; 2017. Disponível em: http://bvsms.saude.gov.br/ bvs/saudelegis/gm/2017/prt2436 2209 2017.ht $\underline{\mathrm{ml}}$

19- Martins AMEBL, Barreto SM, Santos-Neto PE, Sá MAB, Souza JGS, Haikal DS, et al. Greater access to information on how to prevent oral cancer among elderly using primary health care. CiêncSaúdeColetiva2015;20(7):2239-53. DOI: 10.1590/1413-81232015207.15272014

20- Al Sayah F, Williams B, Pederson JL, Majumdar SR, Johnson JA. Health literacy and nurses' communication with type 2 diabetes patients in primary care settings. Nurs Res. 2014;63(6):408-17. DOI: 10.1097/NNR.0000000000000055

21-Paskulin LMG, Bierhals CCBK, Valer DB, Aires $M$, Guimarães NV, Brocker AR, et al. Health literacy of older people in primary care.Acta Paul Enferm.2012;25(nesp1):129-35. DOI: 10.1590/S0103-21002012000800020

22-Edwards M, Wood F, Davies M, Edwards A. 'Distributed health literacy': Longitudinal qualitative analysis of the roles of health literacy mediators and social networks of people living with a long-term health condition. Health Expect. 2015;18(5):1180-93. DOI: 10.1111/hex.12093

23- Silva KM, Santos SMA. The nursing process in family health strategy and the care for the elderly. TextoContexto-Enferm. 2015;24(1):10511. DOI: $10.1590 / 0104-07072015000680013$

24- Abel T,Hofmann K,Ackermann S, Bucher S,Sakarya S. Health literacy among young adults: a short survey tool for public health and health promotion research. Health Promot Int. 2014; 30(3):525-35. DOI: 10.1093/heapro/dat096

25-Wichmann FMA, Couto AM, Areosa SVC, Montañés MCM. Companionship groups as support to improve the health of the elderly. RevBrasGeriatrGerontol. 2013;16(4):821-32. DOI: $\underline{10.1590 / \mathrm{S} 1809-98232013000400016}$

Nota: Este artigo faz parte do Trabalho de Conclusão de Curso de Residência Multiprofissional em Saúde da Família da Universidade Federal do Rio Grande. 
Recebido em:17/05/2018

Aprovado em:10/12/2018

Endereço de correspondência:

Nidia Farias Fernandes Martins

Rua Costa Rica, 737

CEP: 96212-020 - Rio Grande/RS - Brasil

E- mail: nidiaffmartins@gmail.com 